\title{
PROGRAMA DE REABILITAÇÃO CARDÍACA ASSOCIADO AO TREINAMENTO MUSCULAR INSPIRATÓRIO E NA CAPACIDADE FUNCIONAL EM PACIENTES COM INSUFICIÊNCIA CARDÍACA: UMA REVISÃO
}

\author{
HEART REHABILITATION PROGRAM ASSOCIATED WITH INSPIRATORY \\ MUSCLE TRAINING AND FUNCTIONAL CAPACITY IN PATIENTS WITH \\ HEART FAILURE: A REVIEW
}

\author{
Luan Silva Costa ${ }^{1}$ \\ Luciene Pereira Coelho de Azevedo ${ }^{2}$ \\ Islane Cristina Martins ${ }^{3}$ \\ Ítalo gomes da silva ${ }^{4}$
}

RESUMO: Introdução: Insuficiência Cardíaca é uma doença, onde o coração não consegue fornecer sangue suficiente para atender as necessidades metabólicas, causando alterações cardíacas funcionais e estruturais. Com isso, o programa de reabilitação cardíaca, colabora recuperando e preservando a saúde do corpo, com destaque nos exercícios físicos reduzindo as morbimortalidades cardiovasculares. Objetivo: O objetivo do estudo foi formular uma revisão integrativa da literatura a fim de verificar a efetividade de um programa de reabilitação cardíaca associado ao treinamento muscular inspiratório e na capacidade funcional em pacientes com insuficiência cardíaca. Materiais e Métodos: Foram realizadas buscas das referências nas bases de dados: Periódicos CAPES, Google Schorlar, SciELO, Lilacs e Pubmed em julho de 2020. A busca identificou sete artigos que se ajustaram aos critérios estabelecidos. Resultados: De acordo com os resultados encontrados nesta pesquisa foi possível apontar os benefícios demostrados por meio do programa de reabilitação cardíaca como o aumento no condicionamento e na força muscular respiratória, melhor desempenho no teste de caminhada de 6 minutos e elevação dos níveis de aptidão física de pacientes com Insuficiência cardíaca. Conclusão: Conforme os artigos encontrados na literatura consultada, o programa de reabilitação cardíaca é viável, seguro e indicado, devendo ser considerado um recurso importante para tratamento e manutenção da saúde desses pacientes com insuficiência cardíaca , contribuindo nos aspetos físico, psíquico e social, prevenindo complicações, reduzindo as taxas de mortalidade, com isso, melhorando a expectativa de vida.

Palavras-chave: Resultado do tratamento. Reabilitação cardíaca. Terapia respiratória. Teste de esforço. Insuficiência cardíaca.

ABSTRACT: Introduction: Heart failure is a disease, where the heart cannot supply enough blood to meet metabolic needs, causing structural and functional cardiac changes. With this, the cardiac rehabilitation program collaborates in recovering and preserving the health of the body, with emphasis on physical exercises, reducing cardiovascular morbidity and mortality. Objective: The objective of this study was to carry out an integrative literature review in order to verify the effectiveness of a cardiac

\footnotetext{
${ }^{1}$ Bacharel em Fisioterapia - UNIFACOL;

2 Professora do corpo de saúde da UNIFACOL;

3 Doutourando em Neurociências - POSNEURO/UFPE;

4 Bacharel em Fisioterapia - UNIFACOL;
} 
rehabilitation program associated with inspiratory muscle training and functional capacity in patients with heart failure. Materials and Methods: A search was made for references in the CAPES, Google Schorlar, SciELO, Lilacs and Pubmed databases in July 2020. The search allowed the identification of seven articles that met the established criteria. Results: According to the results found in this research, it was possible to point out the benefits shown through the cardiac rehabilitation program, such as increased conditioning and respiratory muscle strength, better performance in the 6-minute walk test and increased levels of physical fitness. of patients with heart failure. Conclusion: According to the literature consulted, the cardiac rehabilitation program is viable, safe and indicated, and should be considered an important resource for the treatment and maintenance of the health of these patients with heart failure, contributing to the physical, psychological and social aspects, preventing complications, reducing mortality rates, thereby improving life expectancy.

Keyword: Treatment outcome. Cardiac rehabilitation. Respiratory therapy. Exercise test. Heart failure.

\section{INTRODUÇÃO}

Atualmente no mundo, as doenças Cardiovasculares (DCVs), são consideradas uma das principais causas de morte (ROTH et al., 2017). Um estudo realizado no Brasil entre 2004 a 20II, demostrou que aproximadamente 8 milhões de pessoas morreram por Insuficiência Cardíaca (IC) (GAUI; KLEIN; DE OLIVEIRA, 20I6).

Nesse sentido, a Insuficiência Cardíaca é uma doença na qual o coração apresenta dificuldade de bombear sangue suficiente para atender as necessidades metabólicas, causando alterações cardíacas funcionais e estruturais, exibindo sinais e sintomas como redução do débito cardíaco e pressões elevadas no repouso ou no esforço, devido a isso, a carência da prática de exercício físico influência bastante no prognostico desses pacientes (ROHDE et al., 2018).

Por isso, as atuais evidências demostram que, a redução da prática de atividade física na IC, promove um desencadeamento de círculo vicioso com efeitos psicológicos negativos, contribuindo no aumento dos sintomas e intolerância ao exercício, causando a redução da capacidade funcional (SOKORELI et al., 2016).

Assim sendo, pacientes diagnosticados com IC tem uma redução da sua capacidade funcional aeróbica, ocasionado por disfunções da musculatura respiratória. A atividade física nesses pacientes é limitada, onde acarreta fadiga, dispneia e fraqueza muscular inspiratória, limitando a realização de exercícios, causando cada dia mais uma piora da qualidade de vida (ANDRADE et al., 2020).

Desse modo, a terapia com treinamento muscular inspiratório (TMI) é a mais indicada, pois apresenta uma melhora no consumo de volume máximo de oxigênio $\left(\mathrm{VO}_{2 \max }\right)$, aumentando o desempenho em atividades funcionais e na qualidade de vida (QV), logo, sendo oferecida por meio de programas de reabilitação cardíaca (QV) (METRA; TEERLINK, 20I7). 
Dito isso, de acordo com a Organização Mundial de Saúde (OMS), reabilitação cardíaca é um conjunto de diversas atividades que são necessárias para pacientes cardiopatas melhorar suas condições mentais, físicas e sociais, de maneira que consigam, por meio do seu próprio esforço restaurar suas funções de vida ativa e uma inclusão em meio a sociedade (CASTRO et al., 2005).

Então, o programa de reabilitação cardíaca, contribui recuperando e preservando a saúde do corpo, com destaque nos exercícios físicos reduzindo as morbimortalidade cardiovascular e global e também a taxa de hospitalização (ANDERSON et al., 2016), proporcionando melhoras na aptidão física tanto nas atividades aeróbicas quanto não aeróbico favorecendo equilíbrio, flexibilidade, , força muscular e acréscimo no desempenho de suas atividades de vida diária (LAUKKANEN; KUJALA, 2018).

Vale ressaltar, que reabilitação cardíaca (RC) é indicada para pacientes com disfunções cardíacas, através da intervenção, tem efeitos benéficos na aptidão física, no condicionamento respiratório e na melhora na qualidade de vida, colaborando com a diminuição dos fatores de riscos cardiovasculares (DOBSON et al., 2012; MARTIN et al., 2012; SUNAMURA et al., 2020).

Por isso, o objetivo do estudo foi realizar uma revisão integrativa da literatura a fim de verificar a efetividade de um programa de reabilitação cardíaca associado ao treinamento muscular inspiratório e na capacidade funcional em pacientes com insuficiência cardíaca.

\section{MATERIAIS E MÉTODOS}

Foi feito uma busca na literatura em junho de 2020, nas bases de dados eletrônicas Periódicos CAPES, Google Acadêmico, SciELO, Lilacs e PubMed. Os descritores empregados foram os seguintes: "Resultado do tratamento" AND "Reabilitação cardíaca" AND 'Terapia respiratória" AND "Teste de esforço" AND "Insuficiência cardíaca” e, em inglês, "Treatment outcome" AND "Cardiac rehabilitation" AND "Respiratory therapy" AND "Exercise test" AND "Heat failure" em todas as bases de dados. Foram selecionados o7 artigos, sendo incluídos os que se ajustaram segundo os critérios de elegibilidade conforme a Figura I. Os critérios de inclusão foram: trabalhos acadêmicos (monografia, tese, dissertação) artigos nos idiomas inglês, espanhol e português, nos últimos cinco anos, envolvendo a efetividade de um programa de reabilitação cardíaca associado ao treinamento muscular inspiratório e na capacidade funcional em pacientes com insuficiência cardíaca. Os critérios de exclusão foram estudos que apresentassem fuga de tema, artigos de revisão de literatura ou metanálise e artigos duplicados. 
Revista Ibero- Americana de Humanidades, Ciências e Educação- REASE

Figura 1. Fluxograma de seleção e inclusão dos artigos

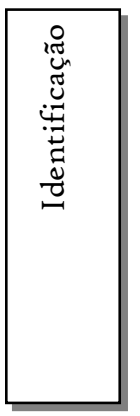

Artigos encontrados nas bases de dados dos periódicos capes, google acadêmico, SciELO, Lilacs e PUBMED $(n=19.436)$
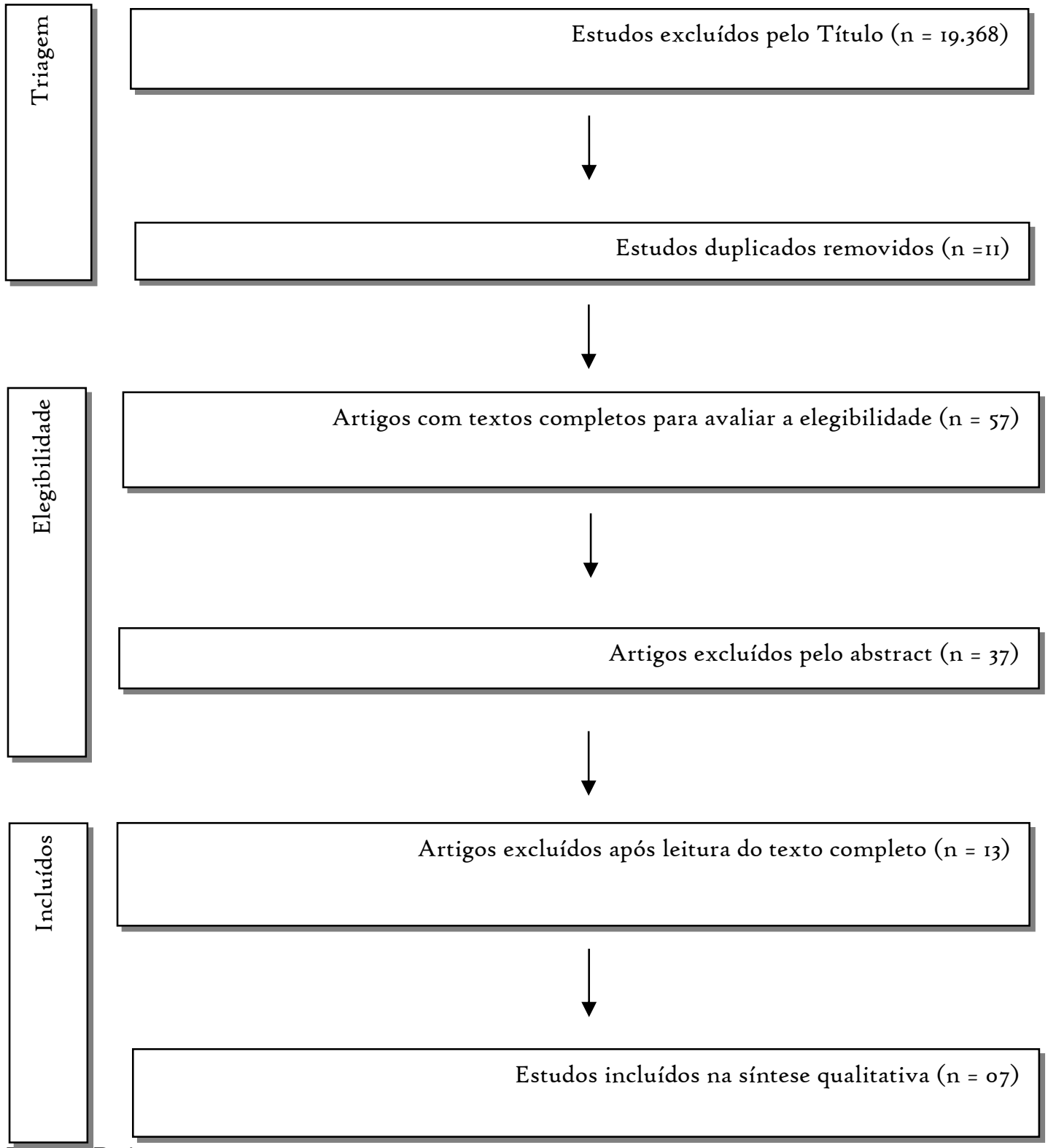

Fonte: Próprio autor

\section{RESULTADOS}


Os resultados do presente estudo encontram-se no Quadro I.

Quadro I - Caracterização dos artigos que compõe a Revisão Integrativa.

\begin{tabular}{|c|c|c|c|c|c|c|}
\hline $\mathbf{N}$ & Data & Título & Autores & Periódico & Objetivos & Resultados \\
\hline I & 2020 & $\begin{array}{l}\text { Diretriz } \\
\text { Brasileira de } \\
\text { Reabilitação } \\
\text { Cardíaca }\end{array}$ & $\begin{array}{l}\text { CARVALHO } \\
\text { T. DE et al }\end{array}$ & $\begin{array}{l}\text { Sociedade } \\
\text { Brasileira de } \\
\text { Cardiologia. }\end{array}$ & $\begin{array}{lr}\text { Descrever } & \text { as } \\
\text { normas } & \text { que } \\
\text { devem } & \text { ser } \\
\text { seguidas em um } \\
\text { programa de } \\
\text { Reabilitação } \\
\text { Cardiovascular } \\
\text { (RCV), a função } \\
\text { de cada } \\
\text { profissional da } \\
\text { equipe, o que deve } \\
\text { ser feito } \\
\text { decorrente de cada } \\
\text { patologia dos } \\
\text { pacientes } \\
\text { cardiopatas } \\
\text { demostrar } \\
\text { evidências as } \\
\text { cientificas dos } \\
\text { estudos atuais. }\end{array}$ & $\begin{array}{l}\text { A RCV } \\
\text { proporcionou altos } \\
\text { níveis de aptidão } \\
\text { física passíveis de } \\
\text { obtenção, de modo } \\
\text { a reduzir o risco de } \\
\text { casos } \\
\text { cardiovasculares e } \\
\text { fornecer pela } \\
\text { benefícios a serem } \\
\text { auferidos prica regular de } \\
\text { prática } \\
\text { atividades físicas, } \\
\text { culminando com a } \\
\text { diminuição da } \\
\text { mortalidade geral. }\end{array}$ \\
\hline 2 & 2018 & $\begin{array}{l}\text { Efeitos de um } \\
\text { programa de } \\
\text { reabilitação } \\
\text { cardíaca na } \\
\text { força de } \\
\text { pacientes com } \\
\text { insuficiência } \\
\text { cardíaca: um } \\
\text { estudo piloto }\end{array}$ & $\begin{array}{l}\text { Amanda } \\
\text { Rafaely } \\
\text { Baldoino }\end{array}$ & $\begin{array}{l}\text { TCC } \\
\text { apresentado } \\
\text { ao programa } \\
\text { de graduação } \\
\text { da } \\
\text { Universidade } \\
\text { de Brasília - } \\
\text { UnB. }\end{array}$ & $\begin{array}{l}\text { Investigar o efeito } \\
\text { de programas de } \\
\text { RC, resistido em } \\
\text { circuito (TRC) e } \\
\text { aeróbio de alta } \\
\text { intensidade } \\
\text { (HIT) na força } \\
\text { de preensão } \\
\text { palmar } \\
\text { periférica } \\
\text { pacientes de } \\
\text { ICC. }\end{array}$ & 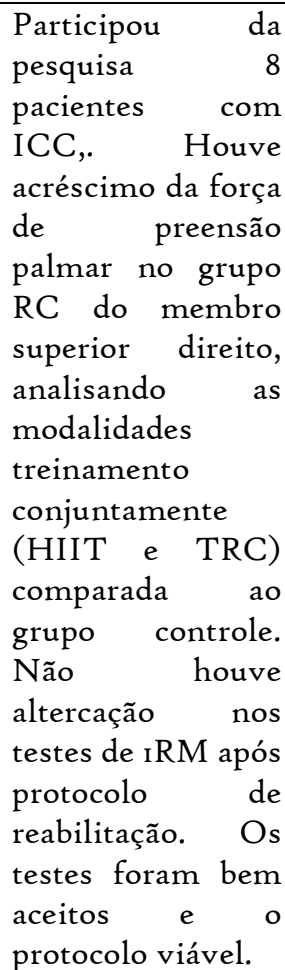 \\
\hline 3 & 2017 & $\begin{array}{l}\text { Força } \\
\text { muscular } \\
\text { inspiratória } \\
\text { de pacientes } \\
\text { com }\end{array}$ & $\begin{array}{l}\text { Thaís Galvão } \\
\text { Araújo; }\end{array}$ & $\begin{array}{l}\text { TCC } \\
\text { apresentado } \\
\text { ao programa } \\
\text { de graduação } \\
\text { da } \\
\text { Universidade }\end{array}$ & $\begin{array}{l}\text { Comparar os } \\
\text { valores da PImáx } \\
\text { obtidos por meio } \\
\text { do } \\
\text { manovacuômetro } \\
(\mathrm{MV}) \text { e do }\end{array}$ & $\begin{array}{l}\text { A média aferida da } \\
\text { PImáx através do } \\
\text { manovacuômetro } \\
\text { quando } \\
\text { comparada com a } \\
\text { PImáx do PB foi }\end{array}$ \\
\hline
\end{tabular}




\begin{tabular}{|c|c|c|c|c|c|c|}
\hline $\mathbf{N}$ & Data & Título & Autores & Periódico & Objetivos & Resultados \\
\hline & & $\begin{array}{l}\text { insuficiência } \\
\text { cardíaca } \\
\text { chagásica: } \\
\text { análise entre } \\
\text { duas } \\
\text { metodologias } \\
\text { de avaliação }\end{array}$ & & $\begin{array}{l}\text { de Brasília - } \\
\text { UnB. }\end{array}$ & $\begin{array}{l}\text { POWERBreathe }{ }^{\circledR} \\
\text { (PB) os pacientes } \\
\text { com IC. }\end{array}$ & $\begin{array}{l}\text { significativamente } \\
\text { maior. Observou- } \\
\text { se uma forte } \\
\text { correlação entre a } \\
\text { PImáx MV e a } \\
\text { PImáx PB nos } \\
\text { pacientes } \\
\text { ICC. }\end{array}$ \\
\hline 4 & 2018 & $\begin{array}{l}\text { Safety and } \\
\text { Efficacy of } \\
\text { Aerobic } \\
\text { Exercise } \\
\text { Training } \\
\text { Associated to } \\
\text { Non-Invasive } \\
\text { Ventilation } \\
\text { in Patients } \\
\text { with Acute } \\
\text { Heart Failure }\end{array}$ & $\begin{array}{l}\text { Mayron F. } \\
\text { Oliveira; Rita } \\
\text { C. Santos; } \\
\text { Suellen A. } \\
\text { Artz; } \\
\text { Vanessa M. F. } \\
\text { Mendez; } \\
\text { Denise M. L. } \\
\text { Lobo; Edileide } \\
\text { B. Correia; } \\
\text { Almir S. } \\
\text { Ferraz; } \\
\text { Iracema I. K. } \\
\text { Umeda;. }\end{array}$ & $\begin{array}{l}\text { Sociedade } \\
\text { Brasileira de } \\
\text { Cardiologia. }\end{array}$ & 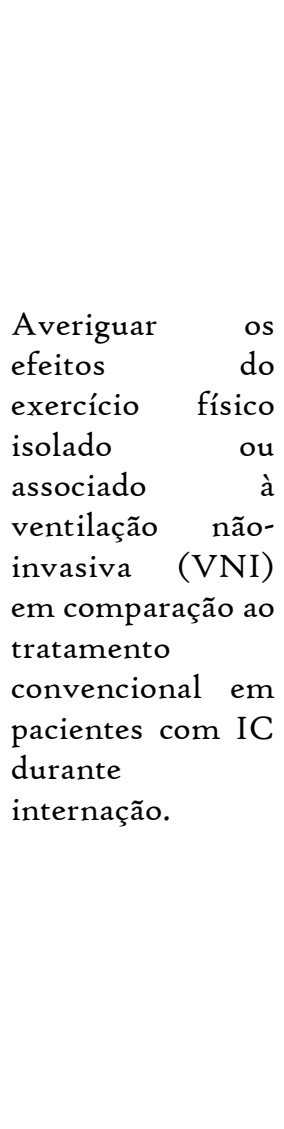 & 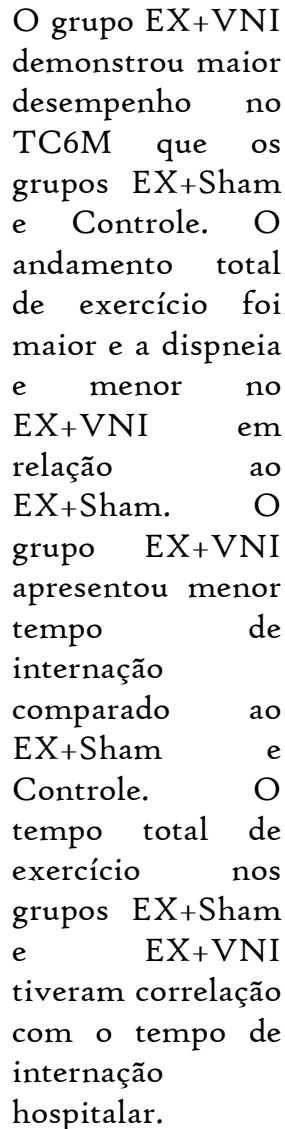 \\
\hline
\end{tabular}




\begin{tabular}{|c|c|c|c|c|c|c|}
\hline $\mathrm{N}$ & Data & Título & Autores & Periódico & Objetivos & Resultados \\
\hline 5 & 2017 & $\begin{array}{l}\text { Eficácia e } \\
\text { segurança do } \\
\text { treinamento } \\
\text { intervalado } \\
\text { de alta } \\
\text { intensidade } \\
\text { em pacientes } \\
\text { com } \\
\text { insuficiência } \\
\text { cardíaca }\end{array}$ & $\begin{array}{l}\text { Lilian } \\
\text { pereira } \\
\text { verardo }\end{array}$ & $\begin{array}{l}\text { Dissertação } \\
\text { apresentada } \\
\text { ao curso de } \\
\text { mestrado } \\
\text { da UFMG. }\end{array}$ & $\begin{array}{l}\text { Aferir } \\
\text { comparar os } \\
\text { resultados } \\
\text { na } \\
\text { capacidade } \\
\text { funcional do } \\
\text { treinamento } \\
\text { intervalado } \\
\text { de alta } \\
\text { intensidade } \\
\text { em relação } \\
\text { ao } \\
\text { treinamento } \\
\text { contínuo de } \\
\text { moderada } \\
\text { intensidade } \\
\text { em } \\
\text { indivíduos } \\
\text { com IC. }\end{array}$ & $\begin{array}{l}\text { Observou um acréscimo } \\
\text { na distância do teste de } \\
\text { caminhada (ISWT) para } \\
\text { osdois grupos. No } \\
\text { questionário de atividade } \\
\text { física (DASI), a maior } \\
\text { parte dos indivíduos, } \\
\text { para ambos os grupos, } \\
\text { apresentaram um } \\
\text { aumento no escore, o que } \\
\text { constitui que eles foram } \\
\text { capazes de realizar um } \\
\text { maior número de } \\
\text { atividades após o } \\
\text { tratamento. Da mesma } \\
\text { forma, visualizou-se que } \\
\text { no questionário de } \\
\text { qualidade de vida } \\
\text { (MLHFQ), que a } \\
\text { maioria dos indivíduos, } \\
\text { para os dois grupos, } \\
\text { tiveram uma redução do } \\
\text { escore, indicando uma } \\
\text { melhora na qualidade } \\
\text { desses indivíduos após o } \\
\text { tratamento. Os grupos } \\
\text { exibiram melhora nos } \\
\text { aspectos analisados. } \\
\text { Todos os pacientes } \\
\text { envolvidos no estudo } \\
\text { existiram resposta } \\
\text { positiva em todas as } \\
\text { variáveis, e qualquer } \\
\text { evento adverso duranteo } \\
\text { treinamento }\end{array}$ \\
\hline
\end{tabular}




\begin{tabular}{|c|c|c|c|c|c|c|}
\hline & & & & & & $\begin{array}{l}\text { identificado. Esses } \\
\text { resultados informam que } \\
\text { não há uma inferioridade } \\
\text { do TIAI, ou que o } \\
\text { mesmo traga prejuízo } \\
\text { para o indivíduo. }\end{array}$ \\
\hline 6 & 2016 & $\begin{array}{l}\text { Reabilitação } \\
\text { cardíaca fase } \\
\text { III associada } \\
\text { à VNI no } \\
\text { tratamento } \\
\text { da ICC: um } \\
\text { estudo de } \\
\text { caso }\end{array}$ & $\begin{array}{l}\text { Eveline } \\
\text { Maria } \\
\text { Calixtre; } \\
\text { Felipe } \\
\text { Alexsandro } \\
\text { Amancio } \\
\text { Prado; Elias } \\
\text { de Almeida; } \\
\text { Gustavo } \\
\text { Mancini } \\
\text { Fontes; } \\
\text { Karoliny } \\
\text { Fernanda } \\
\text { Madureira } \\
\text { da Silva; } \\
\text { Cristiane } \\
\text { Nardi } \\
\text { Gemme; } \\
\text { Susana } \\
\text { Raquel } \\
\text { Rodrigues. }\end{array}$ & $\begin{array}{l}\text { Revista } \\
\text { Saúde e } \\
\text { Meio } \\
\text { Ambiente - } \\
\text { RESMA }\end{array}$ & $\begin{array}{l}\text { Avaliar os } \\
\text { efeitos da } \\
\text { reabilitação } \\
\text { cardíaca fase } \\
\text { III associada } \\
\text { ao uso de } \\
\text { ventilação } \\
\text { não invasiva } \\
\text { no } \\
\text { tratamento } \\
\text { da ICC }\end{array}$ & $\begin{array}{lr}\text { Constatou-se } & \text { uma } \\
\text { redução dos valores de } \\
\text { frequência } & \text { cardíaca, } \\
\text { melhora } & \text { na } \\
\text { funcionalidade } \\
\text { tolerância aos esforços, } \\
\text { aumento na força } \\
\text { muscular respiratória, } \\
\text { acarretando na melhora } \\
\text { da qualidade de vida. }\end{array}$ \\
\hline \multicolumn{7}{|c|}{ Continua } \\
\hline $\mathbf{N}$ & Data & Título & Autores & Periódico & Objetivos & Resultados \\
\hline 7 & 2016 & $\begin{array}{l}\text { Cardiac } \\
\text { rehabilitation } \\
\text { program in } \\
\text { patients with } \\
\text { Chagas heart } \\
\text { failure: a } \\
\text { single-arm } \\
\text { pilot study }\end{array}$ & $\begin{array}{l}\text { Mauro } \\
\text { Felippe } \\
\text { Felix } \\
\text { Mediano; } \\
\text { Fernanda } \\
\text { de Souza } \\
\text { Nogueira } \\
\text { Sardinha } \\
\text { Mendes; } \\
\text { Vivian } \\
\text { Liane } \\
\text { Mattos } \\
\text { Pinto; } \\
\text { Gilberto } \\
\text { Marcelo } \\
\text { Sperandio } \\
\text { da Silva; } \\
\text { Paula } \\
\text { Simplício } \\
\text { da Silva; } \\
\text { Fernanda }\end{array}$ & $\begin{array}{l}\text { Revista da } \\
\text { Sociedade } \\
\text { Brasileira } \\
\text { de } \\
\text { Medicina } \\
\text { Tropical. }\end{array}$ & $\begin{array}{l}\text { investigar os } \\
\text { efeitos da } \\
\text { RC em } \\
\text { pacientes } \\
\text { com ICC }\end{array}$ & $\begin{array}{l}\text { Sete dos } 12 \text { pacientes } \\
\text { incluídos no estudo } \\
\text { completaram o período } \\
\text { de acompanhamento de } 8 \\
\text { meses. Apenas dois } \\
\text { eventos adversos } \\
\text { moderados ocorreram } \\
\text { durante o treinamento } \\
\text { físico. A capacidade } \\
\text { funcional melhorou após } \\
4 \text { meses de RC, enquanto } \\
\text { a fração de ejeção do } \\
\text { ventrículo esquerdo } \\
\text { (FEVE) e a força } \\
\text { respiratória melhoraram } \\
\text { após } 8 \text { meses. Pacientes } \\
\text { com disfunção do } \\
\text { ventrículo direito (VD) } \\
\text { no início do estudo } \\
\text { exibiram uma melhora } \\
\text { na capacidade funcional }\end{array}$ \\
\hline
\end{tabular}




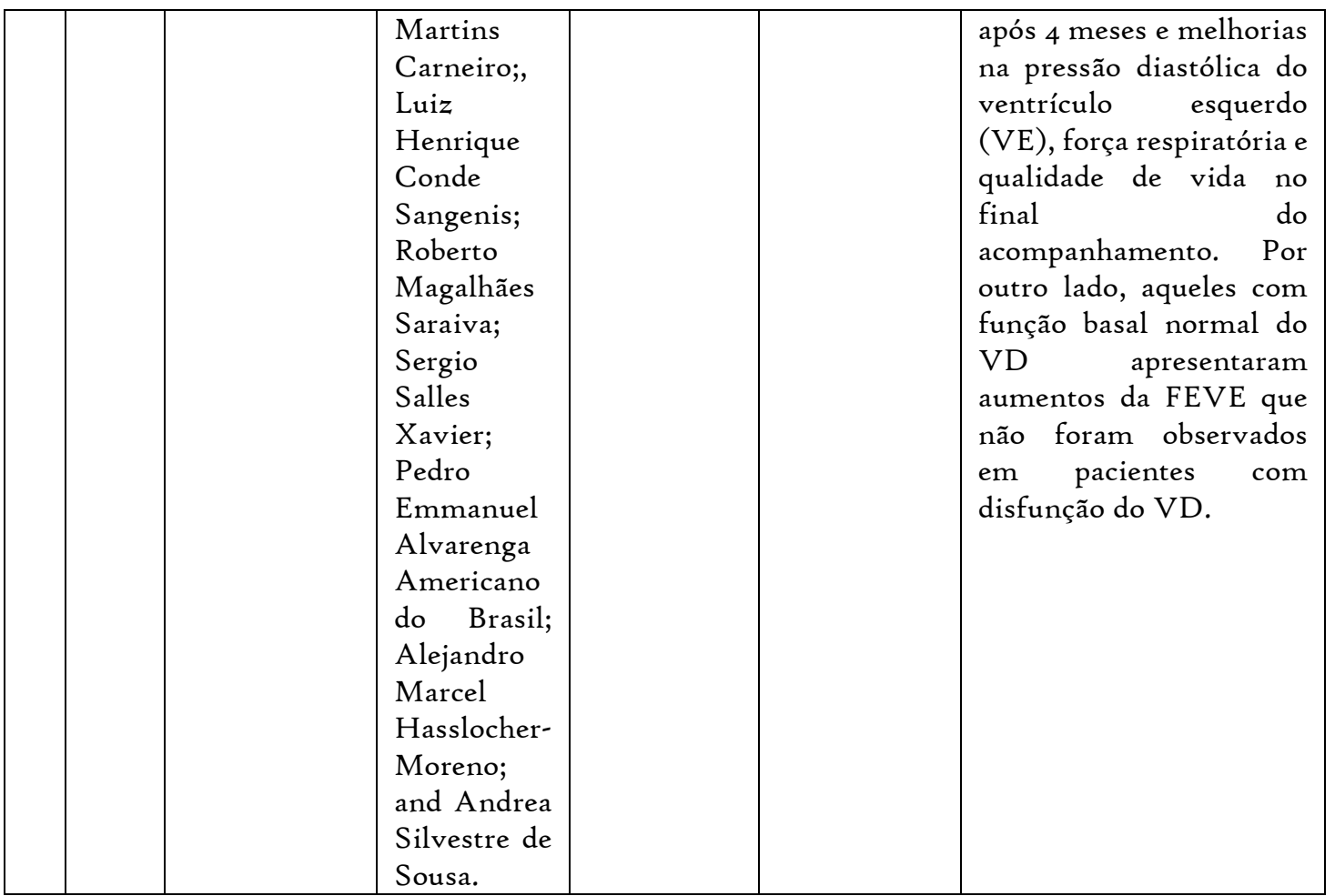

Fonte: Próprio autor

\section{DISCUSSÃO}

O objetivo da referida revisão integrativa foi avaliar a efetividade de um programa de reabilitação cardíaca associado ao treinamento muscular inspiratório e na capacidade funcional em pacientes com insuficiência cardíaca.

Os resultados demostraram que houve efetividade no tratamento de pacientes com insuficiência cardíaca por meio do programa de reabilitação cardíaca onde utilizou-se a ventilação não invasiva (VNI), o treinamento intervalado de alta intensidade (TIAI) e exercícios resistidos, contemplando com melhor desempenho funcional e respiratório nesses pacientes.

Nesse sentido, o paciente com Insuficiência Cardíaca (IC) apresenta disfunção sistólica, com isso é importante a avaliação da força muscular inspiratória e a capacidade funcional. Existem estudos que demostraram valores de pressão inspiratórias até 23\% menores em relação aos indivíduos com função ventricular normal (MEYER et al., 200I).

Para tanto, Araújo, (2017), analisou o valor da pressão inspiratórias máxima (PImáx) mensurada pelo Manovacuômetro e POWERBreathe ${ }^{\circledR}$, observou que para avaliação da força muscular respiratória a mensuração das pressões é influenciada pelo tipo de bocal e sugere a 
padronização desses equipamentos. Pois a diferença nos equipamentos gera discrepâncias nos valores observados.

Ainda, avaliando a PImáx de paciente cardiopatas verificou-se limitações ao realizar exercícios físicos, sendo necessários meios alternativos para auxiliar na melhora do condicionamento desses pacientes, um exemplo seria exercício aeróbico (OLIVEIRA et al., 2018).

Assim, o exercício aeróbico associado a VNI em indivíduos com insuficiência cardíaca aguda ou descompensada é seguro, desde que não apresente sinais ou sintomas de piora durante sua realização, logo, diminui o tempo de internamento em âmbito hospitalar e aumenta a distância do teste de caminhada de o6 minutos (TC6M) (OLIVEIRA et al., 2018).

Isto é, pacientes com IC, onde utilizou-se o Contiunous Positive Airway Pressure (CPAP), todos os dias com duração de mínima de 30 minutos, durante duas semanas antes da prática dos exercícios, observou-se uma melhora na função cardíaca e respiratória (OLIVEIRA et al., 2018).

Além disso, com a pressão positiva ofertada pelo CPAP, ocorre a diminuição da pressão pleural e a redução da pressão interna do ventrículo esquerdo (VE), aumentando a performance contrátil do coração, aumentando o débito cardíaco e reduzindo o volume sistólico final, podendo ser utilizado após os treinos intervalados (IMA et al., 20II).

Nesta sequência, Normandin et al., (2013) comparando o treinamento intervalado com o contínuo em pacientes com IC, notou-se respostas semelhante na capacidade funcional.

Porém, outro estudo demonstra superioridade do TIAI em pacientes com doenças cardiovasculares (DCV) e metabólica para o $\mathrm{VO}_{2}$ pico, nas variáveis como distância percorrida no TC6M e na eficiência ventilatória (VILLELABEITIA JAUREGUIZAR et al., 2016).

Contudo, o treinamento intervalado de alta intensidade complementado com treino de resistência periférica (PRT) e inspiratória (IRT) é benéfico para pacientes com insuficiência cardíaca, pois os indivíduos que participaram do programa elevarão $25 \%$ do $\mathrm{VO}_{2}$ pico comparado ao programa padrão que obtiveram 13\% (HORNIKX et al., 2020; VERARDO, 2017).

Além do mais, os pacientes apresentaram melhoria na capacidade máxima de exercício e ganho na força respiratória periférica e inspiratória, podendo servir como alternativa para um programa de reabilitação cardíaca (HORNIKX et al., 2020).

Não só isso, mas Taylor et al., (2014), analisou 33 estudos relacionados ao treino físico em pacientes com IC predominante de fração de ejeção do ventrículo esquerdo (FEVE) reduzida, 
concluiu que teve redução na taxa de mortalidade, hospitalização e melhora na qualidade de vida comparado ao grupo controle.

Outrossim, Smart; Giallauria; Dieberg, (2013) avaliou II estudos, contendo 287 participantes com IC, sendo 139 do grupo controle e I48 submetidos ao treinamento muscular inspiratório (TMI), observou ganho na eficiência ventilatória, maior distância percorrida no TC6M, na espirometria o aumento da PImáx e melhora na qualidade de vida.

Bem como, Cornelis et al., (2018), observando o treino de exercício para pacientes com IC com fibrilação atrial, relata que deve ser realizada uma avaliação clínica com teste de exercício cardiopulmonar (TECP) antes do início do programa.

Bem como, usar a telemetria para aferir a frequência cardíaca (FC) durante a atividade, e recomenda o treinamento físico de intensidade moderada, pois os resultados mostraram melhora na capacidade aeróbica, no prognostico e na qualidade de vida (CORNELIS et al., 2018).

Isto é, pacientes que possuem Insuficiência Cardíaca Congestiva (ICC) apresentam intolerância a esforços físicos, demostrado através da dispneia e fadiga., podendo melhorar com a pratica de exercícios (COSTA; DE BARROS; LIMA, 20I0).

Assim, o exercício resistido oferece aspecto positivo sobre os fatores de ricos cardiovasculares, sendo favorável na força muscular, capacidade funcional e no bem-estar psicossocial. Isso se deve ao fato do estimulo resultando no aumento da contratilidade na musculatura, promovendo hipertrofia, e melhora na função cardíaca (CALIXTRE et al., 2016).

Nesse sentido, Baldoino, (2018) analisando a força de preensão palmar e periférica de pacientes com IC em um treino aeróbio de alta intensidade (HIIT) e treino resistido em circuito (TRC), verificou o aumento da força de preensão em membro superior direito e não foi observado diferença na força muscular periférica.

Dito isso, a reabilitação cardíaca demonstra ser segura e eficiente para pacientes com IC de alto risco com terapia de ressincronização cardíaca (CRT), os estudos incluídos demostraram que os programas de reabilitação tinham duração de 2 a 4 semanas com frequência de 3 a 5 sessões semanais (CHEN et al., 2019).

Bem como, a prática de exercícios de alta intensidade com até $90 \%$ da frequência cardíaca máxima, respeitando as variáveis de tensão, frequência e duração das atividades. Observou que a reabilitação cardíaca favorece uma melhora no desempenho de exercício, na capacidade funcional e na função cardíaca, contudo estudos futuros são necessários para traduzir essa melhora a longo prazo entre esses pacientes (CHEN et al., 2019). 
Porém, Mediano et al., (2016), relata que após um programa de RC com duração de 8 meses, os pacientes apresentaram uma melhora na função cardíaca ( $17 \%$ na FEVE ), na força muscular respiratória ( $14 \%$ na PImáx e $15 \%$ na PEmáx), e na capacidade funcional ( $11 \%$ no $\mathrm{VO}_{2}$ pico e I9\% no FAI).

Desse modo, é fundamental realizar exercício físicos em pacientes com IC por meio de um programa de RC, trabalhando treinos aeróbicos de moderada e alta intensidade, treinamento da musculatura respiratória e exercício resistido, respeitando sempre os limites funcionais de cada paciente e dando oportunidade aos que estão debilitados e gravemente descondicionado (CARVALHO et al., 2020).

Os resultados encontrados nessa revisão integrativa devem ser utilizados como base para futuras pesquisas, pois os dados obtidos mostram que a prática de atividade física em diversas modalidades é benéfica num programa de reabilitação cardíaca. Dessa forma são necessárias mais divulgações e implementações desses programas que favorecem a população portadores de doenças cardiovasculares. As limitações desse estudo foi a deficiência no quantitativo de artigos relacionados diretamente ao tema abordado.

\section{CONCLUSÃO}

De acordo com a literatura, concluiu-se que existe um resultado positivo observado no coeficiente de frequência cardíaca (FC), fração de ejeção do ventrículo esquerdo (FEVE), capacidade funcional, força muscular respiratória, tolerância aos esforços e refletindo na melhora da qualidade de vida, o que contribui nos aspetos físico, psíquico e social.

Desse modo, o programa de reabilitação cardíaca é viável, seguro e indicado, devendo ser considerado um recurso importante para tratamento e manutenção da saúde desses pacientes com insuficiência cardíaca, além de prevenir possíveis complicações, reduzindo os índices de morbidade e mortalidade, com isso, melhorando a expectativa de vida.

Referências

ANDERSON, L. et al. Exercise-Based Cardiac Rehabilitation for Coronary Heart Disease Cochrane Systematic Review and Meta-Analysis. Journal of the American College of Cardiology, v. 67, n. I, p. I-12, 2016.

ANDRADE, C. C. F. DE et al. Treinamento Muscular Inspiratório Como Recurso Para a Proteção Do Indivíduo Com Insuficiência Cardíaca. Psicologia e Saúde em Debate, v. 6, n. I, p. 49-59, 2020.

ARAÚJO, T. G. Força muscular inspiratória de pacientes com insuficiência cardíaca chagásica : análise entre duas metodologias de avaliação. 2017. Monografia. (Bacharel 
em Fisioterapia). Universidade de Brasília-UnB, 2017.

BALDOINO, A. R. Efeitos de uma programa de reabilitação cardíaca na força de pacientes com insuficiência cardíaca : um estudo piloto. 2018. Monografia. (Bacharel em Fisioterapia). Universidade de Brasília-UnB, 2018.

CALIXTRE, E. M. et al. Reabilitação cardíaca fase III associada à VNI no tratamento da ICC : um estudo de caso. Revista Saúde e Meio Ambiente - RESMA, v. 3, n. 2, p. 62-76, 2016.

CARVAlHO, T. DE et al. Diretriz Brasileira de Reabilitação Cardiovascular - 2020. Arquivos Brasileiros de Cardiologia, v. II4, n. 5, p. 943-987, 2020.

CASTRO, R. R. T. DE et al. Diretriz de Reabilitação Cardíaca. Arquivos Brasileiros de Cardiologia, v. 84, n. 5, p. 431-440, 2005.

CHEN, Z. B. et al. Meta-analysis of the effects of cardiac rehabilitation on exercise tolerance and cardiac function in heart failure patients undergoing cardiac resynchronization therapy. BioMed Research International, v. 2019, p. 8-16, 2019.

CORNELIS, J. et al. Exercise Training in Heart Failure Patients With Persistent Atrial Fibrillation: a Practical Approach. Cardiac Failure Review, v. 4, n. 2, p. IO7-III, 2018.

COSTA, M. F. DE L. E; DE BARROS, M. P.; LIMA, J. H. M. The impact of continuous positive airway pressure (CPAP) on the cardiac rehabilitation of patients with congestive heart failure: Case report. Arquivos Brasileiros de Cardiologia, v. 95, n. I, p. 2009-2011, 2010.

DOBSON, L. E. et al. Is cardiac rehabilitation still relevant in the new millennium? Journal of Cardiovascular Medicine, v. 13, n. I, p. 32-37, 2012.

GAUI, E. N.; KLEIN, C. H.; DE OLIVEIRA, G. M. M. Proportional mortality due to heart failure and ischemic heart diseases in the Brazilian regions from 2004 to 2011. Arquivos Brasileiros de Cardiologia, v. 107, n. 3, p. 230-238, 2016.

HORNIKX, M. et al. Effectiveness of high intensity interval training supplemented with peripheral and inspiratory resistance training in chronic heart failure: a pilot study. Acta Cardiologica, v. 75, n. 4, p. 339-347, 2020.

IMA, E. DA S. et al. Suporte ventilatório na capacidade funcional de pacientes com insuficiência cardíaca: estudo piloto. Arquivo Brasileiro de Cardiologia, v. 96, n. 3, p. 227-232, 2011.

LAUKKANEN, J. A.; KUJALA, U. M. Low Cardiorespiratory Fitness Is a Risk Factor for Death: Exercise Intervention May Lower Mortality? Journal of the American College of Cardiology, v. 72, n. 19, p. 2293-2296, 2018. 
MARTIN, B. J. et al. Cardiac rehabilitation attendance and outcomes in coronary artery disease patients. Circulation, v. 126, n. 6, p. 677-687, 2012.

MEDIANO, M. F. F. et al. Cardiac rehabilitation program in patients with chagas heart failure: A single-arm pilot study. Revista da Sociedade Brasileira de Medicina Tropical, v. 49, n. 3, p. 319-328, 2016.

METRA, M.; TEERLINK, J. R. Heart failure. The Lancet, v. 390, n. IoIo6, p. 198I1995, 2017.

MEYER, F. J. et al. Respiratory muscle dysfunction in congestive heart failure: Clinical Correlation and Prognostic Significance. Circulation, v. 103, n. 17, p. 2153-2158, 2001.

NORMANDIN, E. et al. Acute Responses to Intermittent and Continuous Exercise in Heart Failure Patients. Canadian Journal of Cardiology, v. 29, n. 4, p. 466-471, 2013.

OLIVEIRA, M. F. et al. Safety and efficacy of aerobic exercise training associated to non-invasive ventilation in patients with acute heart failure. Arquivos Brasileiros de Cardiologia, v. IIo, n. 5, p. 467-475, 2018.

ROHDE, L. E. P. et al. Diretriz brasileira de insuficiência cardíaca crônica e aguda. Arquivos Brasileiros de Cardiologia, v. III, n. 3, p. 436-539, 2018.

ROTH, G. A. et al. Global, Regional, and National Burden of Cardiovascular Diseases for ro Causes, 1990 to 2015. Journal of the American College of Cardiology, v. 70, n. I, p. $1-25,2017$.

SMART, N. A.; GIALLAURIA, F.; DIEBERG, G. Efficacy of inspiratory muscle training in chronic heart failure patients: A systematic review and meta-analysis. International Journal of Cardiology, v. 167, n. 4, p. 1502-1507, 2013.

SOKORELI, I. et al. Depression as an independent prognostic factor for all-cause mortality after a hospital admission for worsening heart failure. International Journal of Cardiology, v. 220, n. I, p. 202-207, 2016.

SUNAMURA, M. et al. Patients who do not complete cardiac rehabilitation have an increased risk of cardiovascular events during long-term follow-up. Netherlands Heart Journal, v. 28, n. 9, p. 46o-466, 2020.

TAYLOR, R. et al. Exercise based rehabilitation for heart failure. Cochrane Database of Systematic Reviews, n. 4, p. I-I25, 2014.

VERARDO, L. P. Eficácia e segurança do treinamento intervalado de alta intensidade em pacientes com insuficiência cardíaca. 2017. Dissertação. (Mestrado). Universidade Federal de Minas Gerais, 2017. 
VILLELABEITIA JAUREGUIZAR, K. et al. Effect of High-Intensity Interval Versus Continuous Exercise Training on Functional Capacity and Quality of Life in Patients with Coronary Artery Disease: A randomized clinical trial. Journal of Cardiopulmonary Rehabilitation and Prevention, v. 36, n. 2, p. 96-105, 2016. 